\title{
Can we quantify the risk of embolization for a free-floating thrombus?
}

\author{
Ruggero De Paulis, MD, and Luca Weltert, MD
}

\author{
From the Department of Cardiac Surgery, European Hospital, Rome, Italy. \\ Disclosures: Authors have nothing to disclose with regard to commercial support. \\ Received for publication Dec 20, 2016; accepted for publication Dec 27, 2016; available ahead of print Jan 19, \\ 2017 \\ Address for reprints: Ruggero De Paulis, MD, Cardiac Surgery Department, European Hospital, Via Portuense \\ 700, 00151 Rome, Italy (E-mail: rdepaulis58@gmail.com). \\ J Thorac Cardiovasc Surg 2017;153:804-5 \\ $0022-5223 / \$ 36.00$ \\ Copyright (c) 2017 by The American Association for Thoracic Surgery \\ http://dx.doi.org/10.1016/j.jtcvs.2016.12.025
}

The high resolutions of various imaging techniques, from echocardiography to computed tomography, have enormously increased our ability to detect, visualize and characterize a variety of abnormal tissues that can adhere to the inner layer of the aorta. In most cases, these findings are due to atherosclerotic plaque or stratified thrombus. It is well known how thrombus may progressively stratify in area of partial flow stagnation like aneurysmal portion of the aortic wall or portion of marked curvature or bending. On the other hand, the high velocity of the blood inside a high-pressure system such as the aorta usually prevents the formation and buildup of material partially attached to the wall and floating into the bloodstream.

Nevertheless, there are some clinical and pathologic conditions, such as abnormal coagulation factors, malignancy, steroids, and trauma, that may alter the normal cascade of event and cause the formation of pedunculated thrombi. In this regard, the article in this issue of the Journal by Yang and colleagues ${ }^{1}$ shows how the use of endovascular procedures may be another potential cause of thrombus formation to the inner surface of the prosthesis, either at their interface with the host aortic wall or at the junction between adjacent segments of the prosthesis.

Free-floating thrombus in the ascending aorta, aortic arch, or descending aorta is such a rare entity that no actual coded guidelines exist to help physicians in their decisionmaking process. Nevertheless, the rapidly increasing prevalence of great vessel stenting procedures in the last 5 years establish the ground for this kind of lesion to become much more frequent.

The study by Yang and colleagues ${ }^{1}$ is an almost anecdotal review of their personal experience and of the available clinical literature. In this respect, the article might be considered to carry the lowest level of scientific evidence, with a slim chance of finding a place in a high-quality scientific journal. The urge for a better understanding is so clearly pointed out, however, that this study represents a message to the scientific community to collect more cases pathology. largely unexplored.

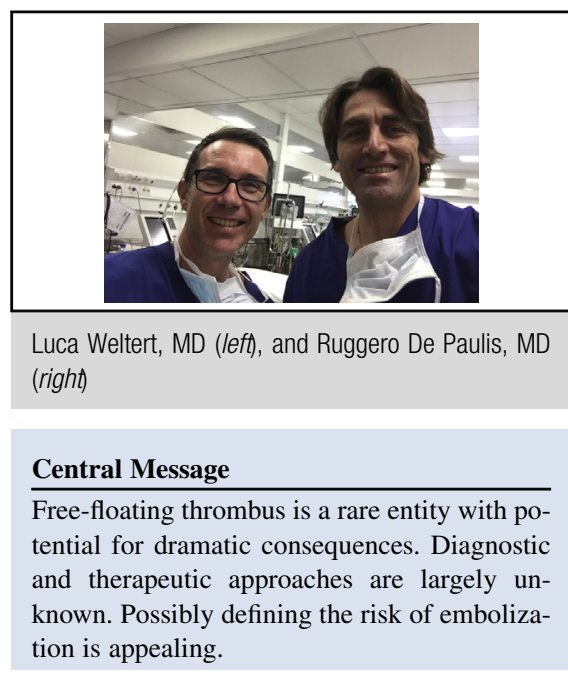

See Article page 791 .

and build up more experience to deal with such

The key intuition is the creation of an index to predict the clinical evolution of a free-floating thrombus. Will it break, with dramatic embolic consequence? Will it react positively to prolonged anticoagulant therapy? Should the physician embrace the risk of a major surgical operation? Would an endovascular approach be appropriate? All these questions remain unresolved, and the therapeutic approach here is

Yang and colleagues ${ }^{1}$ here advocate the creation of a new index, the break-off risk ratio, defined as the ratio between the lengths of the floating and attached portions. The idea is attractive, because it produces a simple, reproducible number that carries a common sense--guided direct correlation with the expected risk. As often is the case in complex biologic environments, many more variables probably play some role in the determinism of the condition, included lesion location, morphology, size, the involved portion of the aorta, concomitant visceral or vascular embolism, the presence of rigid stent, or the dynamic changes of these signs during the follow-up. Everyday experience shows that simple models tend to be rapidly adopted and easily evaluated, however, and thus they could form the basis for a rapid revision as more data are collected. Are we not still posing a surgical indication for an ascending aorta aneurysm on the basis of a simple transverse diameter, when we know that many other factors are involved ${ }^{2}$ On the 
other hand, the study is extremely limited in case number, thus nullifying its inferential significance, and the breakoff risk ratio index should definitely be evaluated further on larger caseloads. The day that we have a reliable index or a shared approach to deal with this rare condition, we can reconsider the significance of these preliminary observations, as well as the efforts toward a scientific standardization of the diagnostic and therapeutic approaches.

\section{References}

1. Yang S, Yu J, Zeng W, Yang L, Teng L, Cui Y, et al. Aortic floating thrombus de tected by computed tomography angiography incidentally: five cases and a literature review. J Thorac Cardiovasc Surg. 2017;153:791-803.

2. Erbel R, Aboyans V, Boileau C, Bossone E, Bartolomeo RD, Eggebrecht H, et al ESC Committee for Practice Guidelines. 2014 ESC Guidelines on the diagnosis and treatment of aortic diseases: document covering acute and chronic aortic diseases of the thoracic and abdominal aorta of the adult. The Task Force for the Diagnosis and Treatment of Aortic Diseases of the European Society of Cardiology (ESC). Eur Heart J. 2014;35:2873-926. Erratum in: Eur Heart J. 2015;36:2779. 